inheritance; 3) loss of tendon reflexes in lower limbs; 4) dysarthria; and 5 ) posterior column signs. The more benign 'Acadian' subtype (Barbeau et al, 1984) has previously been differentiated from the classical, French and French Canadian, type of FA and has been attributed to a mutation at the same locus (Keats BJ, Chamberlain S et al. Am I Med Genet 1989;33:266).

\title{
LORENZO OIL THERAPY FOR ADRENOLEUKODYSTROPHY
}

Dietary therapy with glycerol trioleate and glycerol trierucate (Lorenzo oil) was tested in 108 adult patients with adrenomyeloneuropathy phenotype of adrenoleukodystrophy (ALD) at Johns Hopkins Hospital and the Kennedy Krieger Institute, Baltimore, MD. Pattern-reversal visual evoked potentials were used to evaluate visual pathways before and after treatment for 1 year. Very-long-chain fatty acid (VLCFA) levels were markedly reduced, but visual evoked potentials remained abnormal or became abnormal. No patients improved, and there was no evidence that reduction in VLCFA levels benefited or retarded demyelination of visual pathways. (Kaplan PW, Moser HW et al. Visual evoked potentials in adrenoleukodystrophy: a trial with glycerol trioleate and Lorenzo oil. Ann Neurol Aug 1993; $\underline{34: 169-174) .(R e s p o n d: ~ D r ~ K a p l a n, ~}$ Department of Neurology, Francis Scott Key Medical Center, 4940 Eastern Avenue, Baltimore, MD 21224).

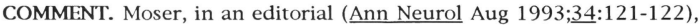
reviews the development of therapies for adrenoleukodystrophy and describes Lorenzo oil as "a prematurely amplified hope." Trials of the oil in Europe and the USA have failed to demonstrate a significant effect on the rate of progression of the childhood cerebral form of ALD. A possible preventive effect of Lorenzo oil in patients who have not yet developed the neurological disability is under investigation. Of 61 asymptomatic patients treated for a few months up to 4 years at the Kennedy Krieger Institute, one has developed childhood cerebral ALD and 7 have shown progressive demyelination on the MRI. Longer follow-up is required to determine the outcome in the 53 who remain asymptomatic. A depression in platelets was an unexpected side effect of Lorenzo oil therapy.

\section{METACHROMATIC LEUKODYSTROPHY VARIANTS}

Clinical, pathological, imaging, and genetic findings in a family with multiple allelic mutations of metachromatic leukodystrophy (MLD) are reported from McGill University, Montreal, and McMaster University, Hamilton, Canada. The propositus, a 23-year-old man, presented at age 18 with a 3 -year history of clumsiness and stiffness of gait. Two maternal uncles, ages 48 and 56, were also neurologically impaired. Two siblings, a brother aged 30 